\title{
Direct imaging searches for planets around white dwarf stars
}

\author{
Matt Burleigh ${ }^{1}$, Emma Hogan $^{1}$ and Fraser Clarke ${ }^{2}$ \\ ${ }^{1}$ Department of Physics and Astronomy, University of Leicester, Leicester LE1 7RH, UK \\ email: mbu or eh54 @star.le.ac.uk \\ ${ }^{2}$ Department of Physics, University of Oxford, UK \\ email: fclarke@astro.ox.ac.uk
}

\begin{abstract}
White dwarfs are excellent targets for direct imaging searches for extra-solar planets, since they are up to $10^{4}$ times fainter than their main sequence progenitors, providing a huge gain in the contrast problem. In addition, the orbits of planetary companions that lie beyond the maximum extent of the Red Giant envelope are expected to widen considerably, improving resolution and further encouraging direct detection. We discuss current searches for planetary companions to white dwarfs, including our own "DODO" programme. At the time of writing, no planetary companion to a white dwarf has been detected. The most sensitive searches have been capable of detecting companions $\gtrsim 5 \mathrm{M}_{\mathrm{Jup}}$, and their non-detection is consistent with the conclusions of McCarthy \& Zuckerman (2004), that no more than $3 \%$ of stars harbour $5-10 \mathrm{M}_{\mathrm{Jup}}$ planets at orbits between $75-300 \mathrm{AU}$. Extremely Large Telescopes are required to enable deeper searches sensitive to lower mass planets, and to provide larger target samples including more distant and older white dwarfs. ELTs will also enable spectroscopic follow-up for any resolved planets, and follow-up of any planetary companions discovered astrometrically by GAIA and SIM.
\end{abstract}

Keywords. white dwarfs — planetary systems — stars: low-mass, brown dwarfs infrared: stars

\section{Introduction}

Direct imaging of extra-solar planets around solar-type stars requires expensive and complex technological solutions, one of which, of course, is the future development of Extremely Large Telescopes (ELTs). Current searches employ a combination of coronagraphs and adaptive optics to overcome the twin problems of contrast and resolution, e.g. the Lyot Project (Oppenheimer et al. 2004). However, at the time of writing no planetary companion to a solar-type star has been imaged.

A cheap and sly solution to these problems is simply to ignore main sequence stars and target intrinsically faint stars instead. Several groups are searching for planetary mass companions to very young stars in nearby clusters. Chauvin et al. (2005) have imaged a $\sim 5 \mathrm{M}_{\text {Jup }}$ companion to a young brown dwarf in the TW Hya association (2MASSW J1207334-393254), although the discovery has also ignited a debate as to the exact nature of the object. Is it a true planet that has formed from a circumstellar disk, or should the pair be regarded as a binary brown dwarf?

White dwarfs are another group of intrinsically faint stars suitable for searches for planetary companions. Stellar evolution lends two huge advantages: white dwarfs are up to $\sim 10^{4}$ times fainter than their main sequence progenitors, and the orbits of any planetary companions that lie outside the Red Giant envelope will expand outwards as mass is lost from the central star, increasing the projected star-planet separation. Thus, the problems of contrast and resolution are largely overcome. The evolution of planets in 
the post-main sequence phase is discussed in more detail by Burleigh, Clarke \& Hodgkin (2002) and Debes \& Sigurdsson (2002).

Direct detection of planetary companions to white dwarfs opens up the possibility for spectroscopic investigation of a previously unobserved class of object: mature, old gas giants or, essentially, $\sim 300-500 \mathrm{~K}$ polytropes (in contrast, the $\sim 10^{6}$ year old $\sim 5 \mathrm{M}_{\text {Jup }}$ companion to 2MASSW J1207 has the spectrum of a late M dwarf). Planetary systems around white dwarfs are of interest in themselves as part of the broader topic of "comparative planetology", i.e. the study of planetary systems in a variety of environments, and for providing information on the final evolutionary stages of solar systems. In addition, many of the white dwarfs most suitable for searches for planetary companions are descended from early-type stars (B, A, and F spectral types) which are not usually the subject of radial velocity searches. By searching for planets around these white dwarfs, we provide information on the frequency of planetary systems around their relatively massive progenitors.

The idea of using white dwarfs to find intrinsically faint, low mass companions is not new. Probst (1983) and Becklin \& Zuckerman (1988) used white dwarfs to search for brown dwarf companions and indeed the latter achieved success with GD 165. More recently, Farihi et al. (2005) have conducted a comprehensive search for brown dwarf companions to several hundred white dwarfs but detected only one new pair (GD 1400, Farihi \& Christopher 2004, Dobbie et al. 2005). We will discuss the various recent direct imaging searches for planetary mass companions to white dwarfs later in this paper, focusing especially on our own project, but mention here should also be made on an indirect detection method, namely searching for timing anomalies in the pulsating ZZ Ceti class of white dwarfs (Mullally et al. 2003), although no planets have so far been found by this method.

\section{The $D O D O$ project}

The opening of access to $8 \mathrm{~m}$ class telescopes, together with new predictions for the evolution and expected luminosities of mature massive Jovian planets (e.g. Burrows et al. 2002), led us in 2002 to initiate a programme to search for such companions to white dwarfs via direct imaging. In particular, we aimed to find planets $>5 \mathrm{M}_{\text {Jup }}$ around white dwarfs $<3$ Gyr old within 20pc. Such companions were predicted to have near-IR magnitudes brighter than $J \sim 24$, commensurate with the expected sensitivity of an $8 \mathrm{~m}$ telescope in a one hour exposure. Brighter companions such as brown dwarfs should be easily detected. Note that the white dwarf age incorporates both the cooling time and the main sequence lifetime of the progenitor.

We christened our project "DODO" - Degenerate Objects around Degenerate Objects (www.star.le.ac.uk/ mbu/planets/index.html). Our target selection criteria gave us a sample of 40 suitable white dwarfs, which we have been observing using Gemini North + NIRI, Gemini South + Flamingos (2002 only), and the VLT + ISAAC (2003 onwards). Note that we do not employ adaptive optics, as we are primarily concerned with searching for the wide companions (generally outside $\sim 2^{\prime \prime}$ from the white dwarf) predicted by the stellar evolution models.

Of course, detection of a 23rd magnitude point source close to a nearby white dwarf does not constitute the direct detection of a planetary companion! Each of our observations contains many such sources, depending on galactic latitude. Our prime discriminator is proper motion, requiring second epoch observations. Since our target white dwarfs are within $20 \mathrm{pc}$, they all have relatively high proper motions, at least $>0.2^{\prime \prime}$ per year. Therefore, we obtain second epoch images one or two years later. 


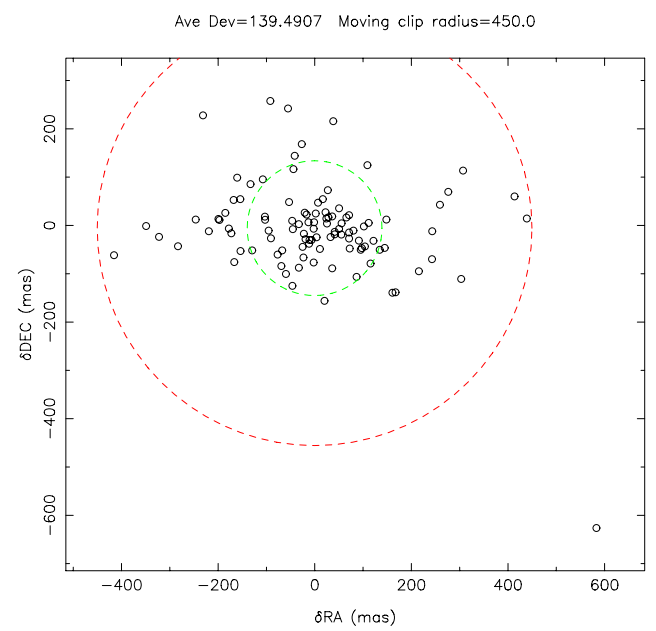

Figure 1. Proper motions of sources detected within the field of WD1647+591, between the 2003 and 2005 observations. The white dwarf has moved $\approx 0.6^{\prime \prime}$ (bottom right). The inner circle illustrates the $1 \sigma$ scatter on the distribution of proper motions, centred on the mean.

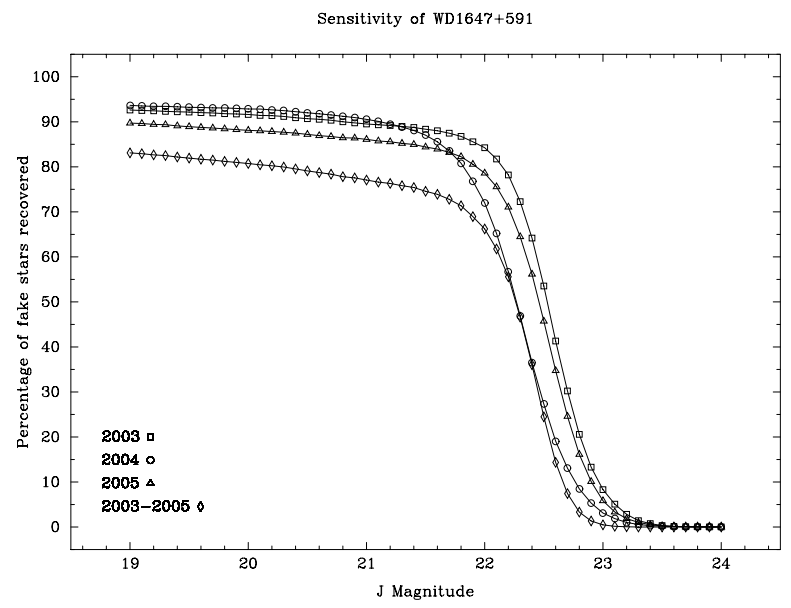

Figure 2. We measure the sensitivity of each observation by injecting fake stars into the images and measuring the number detected by our source search method. $80 \%-90 \%$ are recovered at $J \approx 22$ in the individual observations, and $70 \%$ in both the 2003 and 2005 observations (those used to derive the proper motions in Figure 1). For this white dwarf, a $10 \mathrm{M}_{\mathrm{Jup}}$ companion would be expected to have a brightness $J \approx 19.6$, and a $5 \mathrm{M}_{\text {Jup }}$ companion $J \approx 22.8$.

\section{3. $D O D O$ : results update}

The "DODO" project is reaching maturity. Since 2002 we have observed all of our targets at least once, and $>50 \%$ have second epoch data.

An example of our observing technique and the subsequent data reduction is presented here, for the white dwarf WD $1647+591$. This object was originally observed in May 2003 with NIRI on Gemini North, and again in February 2004. Our analysis of the two images suggested that a faint, $J>22$ object was moving in the field with a very similar proper motion to the white dwarf. If confirmed, this object would have a mass of $\sim 5 \mathrm{M}_{\mathrm{Jup}}$ (from the models of Burrows et al. 2002). 
Table 1. Parameters for WD1647+591 and limits on companions

\begin{tabular}{cc}
\hline Distance & $11 \mathrm{pc}$ \\
Age (cooling + main sequence life) & $0.7 \mathrm{Gyr}$ \\
WD mass & $0.79 \mathrm{M}_{\odot}$ \\
Progenitor mass & $5.0 \mathrm{M}_{\odot}$ \\
Expected magnitude $10 \mathrm{M}_{\mathrm{Jup}}$ companion & $J \approx 19.6$ \\
Expected magnitude $5 \mathrm{M}_{\mathrm{Jup}}$ companion & $J \approx 22.8$ \\
Maximum radius around WD surveyed & $2700 \mathrm{AU}$ \\
Inner search radius & $19 \mathrm{AU}$ \\
\hline
\end{tabular}

To be sure of the reality of this detection, we requested a third epoch observation, which was obtained in February 2005. Unfortunately, the candidate proved to be spurious. Figure 1 shows the proper motions of objects in the $\sim 2^{\prime} \times \sim 2^{\prime}$ field of view of NIRI. The white dwarf's motion is clearly detected $\left(\sim 0.3^{\prime \prime}\right.$ per year $)$, but nothing else is moving with the same motion. We have not tried to subtract the white dwarf's PSF, and we do not attempt to detect objects within $2^{\prime \prime}$ of the star. The search area translates to separations of $20-2700 \mathrm{AU}$, at the distance of the white dwarf $(\approx 11 \mathrm{pc})$. Figure 2 shows the sensitivity of our observations. We randomly injected fake stars into the images and attempted to recover them with the same analysis method used for real sources. A percentage $(\sim 20 \%)$ of the fake stars are never recovered, since they are hidden beneath other objects in the field, or are lost in the low $\mathrm{S} / \mathrm{N}$ edges of the field. However, we recover $\sim 80 \%$ of fake stars to $J \sim 22$. Thereafter, sensitivity rapidly drops off. For comparison, a $5 \mathrm{M}_{\text {Jup }}$ companion to this white dwarf is expected to have a brightness $J=22.8$. The conclusions from our survey for planetary mass companions to WD $1647+591$ are summarised in Table 1 . A paper discussing the analysis of this field, and other early "DODO" results, is currently in preparation (Burleigh et al. 2006).

\section{Do white dwarfs harbour planetary systems?}

The "DODO" project has yet to find any planetary mass companion to a white dwarf, although it is still ongoing. At the time of writing, no other ground-based or space-based search has made a successful detection either, despite the odd false alarm and over-zealous media hype! For example, Friedrich et al. (2005) observed 7 white dwarfs in the Hyades cluster with $H S T$ and NICMOS and found no companions to a limiting mass of $10 \mathrm{M}_{\mathrm{Jup}}$. Debes et al. (2005) found no common proper motion companions $>6 \mathrm{M}_{\mathrm{Jup}}$ at projected separations $>12 \mathrm{AU}$ around the white dwarf G29-38. Several groups have programmes with Spitzer to search for planetary companions as mid-IR photometric excesses (e.g. Hansen et al. 2005). Strictly speaking these are not direct imaging searches, but the mid-IR is in fact the best waveband for such projects. However, no planetary or brown dwarf companion has yet been detected by Spitzer, although debris dust disks have been identified in several cases. These may arise from the tidal disruption of a comet or asteroid that strayed too close to the white dwarf, providing tantalising circumstantial evidence for the existence of old planetary systems (Becklin et al. 2005, Reach et al. 2005).

The failure thus far of any current programme to detect planetary mass companions to nearby white dwarfs does not yet translate to tight limits and conclusions on their frequency, and to the frequency of such objects as companions of the white dwarf progenitors. For that, we must await detailed publication of the results of the ground-based and Spitzer programmes. However, even for the closest, youngest white dwarfs, the current projects are really only sensitive to companions $>5 \mathrm{M}_{\mathrm{Jup}}$, and such objects are already known to be uncommon companions to main sequence stars (at least in orbits $<$ few $\mathrm{AU}$ ). 
It may be that more massive main sequence stars, such as B stars, harbour more massive proto-planetary disks and, therefore, we might expect them and their white dwarf progeny to be more likely to have massive Jovian companions, but this conjecture remains to be proven. Suffice to say, at present the non-detection of planetary mass companions to white dwarfs is consistent with the conclusions of McCarthy \& Zuckerman (2004), that the frequency of $5-10 \mathrm{M}_{\mathrm{Jup}}$ giant planet companions to main sequence stars, orbiting between $75 \mathrm{AU}$ and $300 \mathrm{AU}$, is no more than $\sim 3 \%$.

\section{Future searches}

Clearly, we would like to extend our searches to lower masses, and we would also like to target older and more distant white dwarfs. Both aims simply require larger telescopes and/or more sensitive instruments. The most favourable waveband for direct imaging searches is the mid-IR, and a successful JWST mission will play an important role here (although we emphasise that proper motion is a key discriminator if resolved planetary companions are to be unambiguously detected, and therefore 2 epoch programmes are essential). Ground-based ELTs will similarly be very important in reaching to lower masses and larger sample sizes, most likely in the near-IR $J$ and $H$ bands. We also point out that the GAIA and SIM astrometric surveys should identify white dwarfs with reasonably wide Jovian companions, which could then be resolved by these telescopes, and investigated spectroscopically.

\section{Acknowledgements}

MB acknowledges receipt of a PPARC Advanced Fellowship. EH acknowledges the support of a PPARC Postgraduate Studentship.

\section{References}

Becklin, E.E. \& Zuckerman, B. 1988, Nature 336, 656

Becklin, E.E., Farihi, J., Jura, M. et al. 2005, ApJ 632, L119

Burleigh, M.R., Clarke, F.J. \& Hodgkin S.T. 2002, MNRAS 331, L41

Burleigh, M.R., Clarke, F.J., Hogan, E., Levan, A.J. \& Hodgkin S.T. 2006, MNRAS, in prep.

Burrows, A., Burgasser, A.J., Kirkpatrick, J.D. et al. 2002, ApJ 573, 394

Chauvin, G., Lagrange, A.-M., Dumas, C. et al. 2005, A\&A A 438, L29

Debes, J.H. \& Sigurdsson, S. 2002, ApJ 572, 556

Debes, J.H., Sigurdsson, S. \& Woodgate, B.E. 2005, ApJ 633, 1168

Dobbie, P.D., Burleigh, M.R., Levan, A.J. et al. 2005, MNRAS 357, 1049

Farihi, J. \& Christopher, M. 2004, $A J$ 128, 1868

Farihi, J., Becklin E.E. \& Zuckerman B. 2005, ApJS 161, 394

Friedrich, S., Zinnecker, H., Brandner, W. et al. 2005, in D. Koester and S. Moehler (eds.), 14 th European Workshop on White Dwarfs, (San Francisco: ASP Conference) Series, 334, 431

Hansen, B.M.S., Kulkarni, S. \& Wiktorowicz, S. 2005, astro-ph/0511094

McCarthy, C. \& Zuckerman, B. 2004, AJ 127, 2871

Mullally, F., Mukadam, A., Winget, D.E., Nather, R.E. \& Kepler, S.O. 2003 in D. de Martino, R. Silvotti, J.-E. Solheim \& R. Kalytis (eds.), White Dwarfs, NATO Science Series II Mathematics, Physics and Chemistry, (Dordrecht: Kluwer), 105, 337

Oppenheimer, B.R., Digby, A. P. \& Newburgh, L. et al. 2004, SPIE 5490, 351

Probst, R. 1983, ApJS 53, 335

Reach, W.T., Kuchner, M.J., von Hippel, T. et al. 2005, ApJ 635, L161

\section{Discussion}

ZINNECKER: A comment and a question. The comment is about the non-detection of giant planets around white dwarfs so far. It is quite possible that most planetary companions to 
white dwarfs (with AFG-type main-sequence progenitors) have masses below $3 \mathrm{M}_{J u p}$, so current sensitivity is not good enough to detect these yet. Remember the mass function of planets around solar-type stars increases like $\mathrm{M}_{\text {Planet }}^{-1}$ and less massive planets are much more frequent.

My question is about the Farihi object GD 1400b and its close separation from the white dwarf $(<1 \mathrm{AU})$. This is contrary to the predicted orbit widening due to stellar mass loss. Any idea what's going on there?

BuRleIGH: Jay Farihi obtained a LGS observation of GD 1400 at Keck and the WD/BD pair are unresolved, implying $<1 \mathrm{AU}$ separation. Either this is an unfortunate projection effect and it is wider, or maybe this brown dwarf has survived a phase of common envelope evolution in which it's orbit moved inwards. In fact, we might expect it to be in a very close orbit, although there is no suggestion that this is an accreting CV.

STAPELFELDT: What range of orbital radii (in progenitor system) do your observations probe? This should be bounded by the closest distance to the WD at which you can resolve a separate source, and the outer radius of the field of view of your imager.

BURLEIGH: We typically are only looking outside of a radius of 2 arcsec from the white dwarf. Spitzer will tell us if there are any planets in closer orbits through mid-IR photometric excesses. Depending on its distance, that's a separation of 10-40AU. In the progenitor, that's a few to about 10AU, depending on the progenitor's mass. The FoV of e.g. NIRI on Gemini North is $2^{\prime} \times 2^{\prime}$ with the f16 camera we are using, so again the maximum search radius depends on white dwarf distance, but it is typically a few hundred to one thousand AU. That's great because we expect the planetary orbits to expand in the post-main sequence phase, and you might also expect some dynamical disruption of a multiple-planet system in which some planets are thrown out into very wide, eccentric orbits.

MCCaughrean: You showed that Jupiter-mass planets get very faint at $H$ at 1 Gyr: this is because all the flux has gone out into the thermal IR. So, surely JWST will clean up for these sort of objects.

Burleigh: JWST might find them, that's true. But like Spitzer, will it simply search for photometric excesses and not wider common proper motion companions?

HomeIER: comment: What few detections exist of planets around F-type host stars probably do indicate a trend to higher companion mass for more massive primaries. For more massive progenitors, which lose a large fraction of their mass $(<80 \%)$, is there an upper limit to the initial orbit for which the planet remains bound?

BuRleigh: Don't know, but would be very interested to see the results of such a calculation! 